\title{
A first step towards Quantum Energy Potentials of Electron Pairs
}

\section{Julen Munárriz,,${ }^{1,2}$ Rubén Laplaza, ${ }^{1,2}$ Ángel Martín Pendás, ${ }^{3}$ Julia Contreras-} García $^{2, *}$

${ }^{1}$ Departamento de Química Física and Instituto de Biocomputación y Física de Sistemas Complejos (BIFI), Universidad de Zaragoza, 50009, Zaragoza, Spain

${ }^{2}$ Sorbonne Université, CNRS, Laboratoire de Chimie Théorique, LCT, F. 75005 Paris, France

${ }^{3}$ Laboratorio de Química Física y Analítica, Universidad de Oviedo, 33006, Oviedo, Spain

* Corresponding author: contrera@1ct.jussieu.fr

\begin{abstract}
A first step towards the construction of a quantum force field for electron pairs in direct space is taken. Making use of topological tools (Interacting Quantum Atoms and the Electron Localization Function), we have analysed the dependency of electron pairs electrostatic, kinetic and exchange-correlation energies upon bond stretching. Simple correlations were found, and can be explained by considering electron pairs from the homogeneous electron gas perspective. The model is applicable to various bonding regimes: from homopolar to highly polarized and even to non-conventional bonds. Overall, this is a fresh approach for developing real space-based force fields including an exchangecorrelation term. It provides the relative weight of each of the contributions, showing that in common Lewis structures the exchange correlation term in between electron pairs is negligible. However, our results show that classical approximations progressively fail for delocalized electrons, including lone pairs. This theoretical framework justifies the success of the classic Bond Charge Model approach in solid state systems while it explains the fact that these models are barely used for open structures. Finally, this approach opens the door towards the development of a quantitative energy model based on the ELF topology.
\end{abstract}




\section{Introduction}

The main goal of theoretical chemistry is understanding the inherent link between the atomic composition and observable macroscopic properties. This connection can be easily described in terms of interacting potentials, which directly relate the geometric configuration with the energy of a given system. In the development of such Force Fields (FF), the change in energy upon bond length, angles and torsional angles variation is analyzed and adjusted to reproduce physically sound trends.

This is usually done following simple atomic models. For example, bond lengths are commonly analyzed in terms of Hooke's law, where potential and kinetic energy balances are parametrized to adjust to experimental/computational results. ${ }^{1,2}$ Nonetheless, in many cases, chemical behavior is determined by non-classical terms (e.g. exchange or correlation), which are not easily encoded in these potentials. In order to include this information, one can resort to an accurate quantum description of the system through the Schrödinger equation. However, in quantum terms, the local composition-properties relationship is not bidirectional anymore. The description of a quantum system is global; hence the relationship between the atomic components and the final properties is blurred. Obtaining the properties from the atomic composition can be done right away, yet the arduous inverse connection is still the focus of many inverse design approaches. ${ }^{3}$ Albeit "chemical intuition" can provide a qualitative general picture, and modern wavefunction analysis methods (energetic partitions, topological approaches, etc.) ${ }^{4-6}$ can rationalize specific aspects of chemistry, general bidirectional schemes providing atomic configuration-energy relationships are still scarce. ${ }^{7,8}$ In other words, it is still necessary to develop new models and concepts for understanding the contribution of each atom or functional group in the energy of the system. ${ }^{9}$

a) $\mathrm{H}_{2} \mathrm{O}$

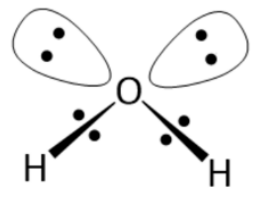

c) $\mathrm{CH}_{3}-\mathrm{NH}_{2}$

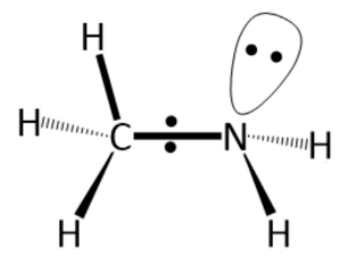

b) $\mathrm{BeCl}_{2}$

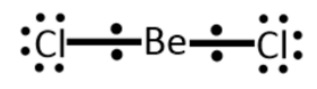

d) $\mathrm{XeF}_{4}$

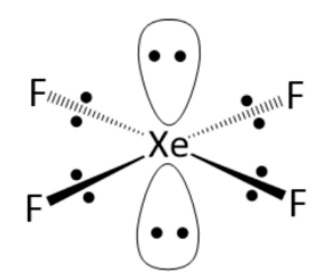

Figure 1. VSEPR geometries for a) $\mathrm{H}_{2} \mathrm{O}$; b) $\mathrm{BeCl}_{2}$; c) $\mathrm{CH}_{3}-\mathrm{NH}_{2}$; and d) $\mathrm{XeF}_{4}$.

\section{I'M NOT SURE THIS FIGURE IS NEEDED}

The problem with atomic models is that they usually require parametrization for different coordination numbers. However, this is not so if we recognize the relevant of the electron pair unit. Before the advent of formal force fields, the use of mere electrostatic reasoning among electron pairs allowed for the first time to predict molecular structure, in 
what is nowadays known as the Valence Shell Electron Pair Repulsion (VSEPR) theory, see Figure $1 .{ }^{10}$ More refined models where kinetic energy is also taken into account were developed afterwards. We postulate that the VSEPR theory can be used as a foundation to quantitatively link compositions and energies in simple terms while including relevant quantum features. The utility of electron pairs as the basis of the representation ${ }^{11,12}$ lies on the fact that paired electrons behave very much like bosons. This means that Pauli correlation is minimized in a system composed by perfectly localized electron pairs, so that the exchange-correlation term (more difficult to disentangle with simple models) is minimal. These advantages have been exploited both in classical approaches, such as the Bond Charge Model (BCM), ${ }^{13,14}$ where only potential and kinetic energy terms are included, as well as in more updated formulations based on quantum mechanical calculations. ${ }^{[9]}$ Of course, we then have to face the identification of electron pairs, and their parametrization. For that, the Interacting Quantum Atoms (IQA) energy decomposition scheme is very suitable, since it allows to obtain and quantify the different energetic contributions from real space partitions of the system. ${ }^{15,16}$ The advantages of the IQA scheme are showcased by its success in the study of different chemical phenomena, such as halogen and hydrogen bonding, ${ }^{17,18}$ and metal-ligand interactions. ${ }^{19}$ IQA is usually coupled to Quantum Theory of Atoms in Molecules (QTAIM) partitions, in order to associate energy terms to atomic contributions. In this sense, it is worth noting the previous work of Popelier et al., who developed the first protein FF based on the QTAIM topology. ${ }^{20}$ This framework also proved to be suitable for geometry optimizations when combined with machine learning approaches. ${ }^{21,22}$ However, since we want to focus on electron pairs, we will couple IQA to the Electron Localization Function (ELF) topology. ${ }^{23}$ The ELF is able to recover Lewis entities, as bonds and lone pairs, providing the classical VSEPR picture, and is thus the perfect starting point for our energy model, allowing to quantify (classic and quantum) interactions between electron pairs. ${ }^{24}$

In this article we propose a first step in constructing a stretching energy model based on the ELF topology. To do so, we evaluate electron pair interactions through classical (kinetic and electrostatic) and quantum energy terms (exchange and correlation). The ease and chemical rigor with which model parameters can be obtained sets the foundations of a fresh approach to real space force fields. In addition, a careful analysis of the relative weight of classical and quantum energy terms is presented. Valuable implications in general force field development are also derived, as an attempt is made to identify the chemical bonds that require quantum terms among those that can be studied under a purely classical perspective. The resulting model is beautifully connected with previous "intuitive" models (e.g. VSEPR ${ }^{1}, \mathrm{BCM}^{25}$ ). It also sets the basis for connecting the ELF topology with the energy of the system, leading to a simple chemical model that attempts to answer the initial question: "how does each atom contribute to the energy of the system?". This knowledge holds the key for the rational design of new materials with desired properties.

\section{Theoretical and computational Methods}

All energy scans and wavefunctions for ELF and IQA analyses were obtained by DFT calculations, using Gaussian 09 program package. ${ }^{26}$ We used B3LYP ${ }^{27,28}$ exchangecorrelation functional in conjunction with $6-31 \mathrm{G}(d)$ basis set. ${ }^{29,30}$ Stability checks were 
carried out with other functionals (PBE and PBE0) ${ }^{31-33}$ as well as Hartree-Focck in conjunction with bigger basis sets $\left(6-311 \mathrm{G}(d, p)\right.$, cc-PVDZ and cc-PVTZ). ${ }^{34}$ Results lead to the same behaviour, obtaining very similar fittings, while the computational cost increased considerably with the increase in the basis set size. These results are provided in Supporting Information. Hence, the small basis set was favoured. Bonding descriptors were computed at the $\mathrm{B} 3 \mathrm{LYP} / 6-311 \mathrm{G}(d, p)$ level to further ensure basis set convergence of the density at the bond critical points.

The partition into localized electron pairs was carried out with the Electron Localization Function (ELF). ${ }^{23}$ The ELF core, $\chi_{\sigma}$, can be interpreted as a measure of the excess of local kinetic energy due to the Pauli principle, relative to the homogeneous electron gas kinetic energy density. ${ }^{35}$ This core is mapped with a Lorentzian function (see eq 1), with values ranging from 0 , when $\chi_{\sigma} \rightarrow \infty$ (in between perfectly localized pairs), to 1 , when $\chi_{\sigma} \rightarrow 0$ (perfectly localized pairs - covalent bonds, lone pairs and internal atomic shells).

$$
\eta(\vec{r})=\frac{1}{1+\chi_{\sigma^{2}}}
$$

The gradient of this function, $\nabla \eta$, is used to induce a topological partition which divides the space into non-overlapping regions (basins) whose properties can be determined by integrating over the associated volume. Hence, if we are interested in, for example, lone pair populations, it suffices to integrate the electron density, $\rho$, over the corresponding region associated to the lone pair maximum.

ELF studies were performed with a locally modified version of the TopMod program ${ }^{36}$ using the monodeterminantal B3LYP/6-31G $(d)$ wavefunctions in conjunction with a tridimensional grid of 200 points in each direction. ELF plots were made with Chimera software. ${ }^{37,38}$

The energies associated with topological basins can be calculated with the Interacting Quantum Atoms (IQA) energy decomposition scheme. ${ }^{39}$ The IQA approach provides unique and rigorous energetic terms that additively recover the exact energy of the system. Additionally, it is solely derived from the wavefunction of the system; hence the error in the total energy is solely determined by the quality with which it was calculated. Unlike many topological analyses, this method is not only suitable for stationary points (e.g. equilibrium geometries), as with virial related energy partitions, but also for non-equilibrium geometries. This feature is crucial for evaluating energy terms along bond elongations in geometry scans.

Energy terms are calculated by partitioning the first and second-order density matrices with respect to real space partitions, usually QTAIM atomic basins. The decomposition for atomic basins $A$ and $B$, is shown in eq 2 .

$$
E=\underbrace{\sum_{A}\left(T_{A}+V_{e n}^{A A}+V_{e n}^{A A}\right)}_{\sum_{A} E_{\text {intra }}^{A}}+\underbrace{\sum_{A>B}\left(V_{e n}^{A B}+V_{n e}^{A B}+V_{n n}^{A B}+V_{e e}^{A B}\right)}_{\sum_{A>B} E_{\text {inter }}^{A B}}
$$

Where $T_{A}$ is the kinetic energy of electrons in atom $\mathrm{A}$ and $V_{e n}^{A B}$ represents the interaction between the electrons in $A$ and the nucleus in $B$. This way, the total energy is divided in a sum of intra and interatomic contributions. Electron-electron interaction can 
be further divided into a classical (electrostatic term) contribution, $V_{\text {elec }}^{A B}$, and a nonclassical, $V_{X C}^{A B}$, which is the sum of exchange $(X)$ and correlation (Corr) terms (see eq 3 ).

$$
V_{\text {ee }}^{A B}=V_{\text {elec }}^{A B}+V_{X C}^{A B}=V_{\text {elec }}^{A B}+V_{X}^{A B}+V_{\text {Corr }}^{A B}
$$

Following this scheme, each contribution to the total energy (intra and interatomic) can be expressed just like in classical approaches, as a sum of kinetic and electrostatic terms, plus a non-classical interatomic term, as shown in eqs 4 and 5 .

$$
\begin{aligned}
& E_{\text {inter }}^{A B}=V_{\text {Coul }}^{A B}+V_{X C}^{A B} \\
& E_{\text {intra }}^{A}=T_{A}+V_{\text {Coul }}^{A A}+V_{X C}^{A A}
\end{aligned}
$$

Where all electrostatic terms have been put under a common "Coul" index: $V_{\text {Coul }}^{A B}=$ $V_{n n}^{A B}+V_{\text {elec }}^{A B}+V_{e n}^{A B}+V_{n e}^{A B}$ and $V_{\text {Coul }}^{A A}=V_{\text {elec }}^{A A}+V_{e n}^{A A}+V_{n e}^{A A}$.

IQA can also be applied to ELF partitions. When considering bonding basins (and valence in general) within the IQA partition, the nuclear terms presented in the previous equations become null $\left(V_{e n}^{B B}=0, V_{n n}^{A B}=0, V_{e n}^{A B}=0\right)$. Therefore, for a bonding basin " $B o n d$ " that interacts with a core basin representing an atom $A$, the different energy terms can be expressed as shown in eqs 6 and 7.

$$
\begin{aligned}
& E_{\text {intra }}^{\text {Bond }}=T_{\text {Bond }}+V_{\text {Coul }}^{\text {Bond }}+V_{X C}^{\text {Bond }} \\
& E_{\text {inter }}^{A-\text { Bond }}=V_{\text {Coul }}^{A-\text { Bond }}+V_{X C}^{A-B o n d}
\end{aligned}
$$

The original IQA implementation could only deal with HF wavefunctions; however, a recent development provides support for DFT-derived ones. ${ }^{16}$ IQA calculations were performed with PROMOLDEN. ${ }^{15,40}$ Since we are interested in developing an energy model accounting for interactions between electron pairs, we coupled IQA with the ELF topology. For this purpose, the original code was modified to perform integration tasks over ELF basins. ${ }^{24}$ The IQA-ELF approach provides an accurate reference that can be used to analyze the behavior of energy terms and for constructing energy potentials that take into account classical as well as non-classical terms. In the next sections, we will examine the dependency of these terms with respect to bond elongation and compare them with previous potentials.

\section{Results and Discussion}

\section{The model}

As a starting point, the model was applied to a series of simple yet representative molecules in the $\mathrm{CH}_{3}-\mathrm{X}\left(\mathrm{X}=\mathrm{CH}_{3}, \mathrm{NH}_{2}, \mathrm{OH}, \mathrm{F}\right)$ series. In order to develop energetic potentials, the $\mathrm{C}-\mathrm{X}$ interatomic distance, $R$, was stretched around the equilibrium position, $R_{e q}$, approximately in the range $[0.90-1.10] R_{e q}$. Since we are resorting to small elongations, the use of a DFT approach is justified. The following standard ELF notation was used: $\mathrm{V}(\mathrm{C}-\mathrm{X})$ represents the $\mathrm{C}-\mathrm{X}$ bond, $\mathrm{V}(\mathrm{X})$ the lone pairs on $\mathrm{X}, \mathrm{C}(\mathrm{Y})$ or simply $\mathrm{Y}$ designates the core of atom $\mathrm{Y}$ and so on. For the sake of simplicity, we will analyze the results for $\mathrm{CH}_{3}-\mathrm{NH}_{2}$ in detail, as an illustrative example with bonds and lone pairs. Similar results have been obtained for the all the molecules in the $\mathrm{CH}_{3}-\mathrm{X}$ test set, and are provided in Supporting Information. 
$\mathrm{CH}_{3}-\mathrm{NH}_{2}$ ELF basins and their populations are shown in Figure 2a. The evolution of ELF populations upon bond stretching (Figure $2 \mathrm{~b}$ ) follows the expected behavior, with a flux of electrons towards the lone pairs as the distance increases. In order to develop working equations for the stretching we need to understand the evolution of the main parameters during this process. Bond charges and bonding basin size were found to change linearly with $R$ (Figures $2 \mathrm{~b}$ and $2 \mathrm{c}$ ). Similar results for the complete $\mathrm{CH}_{3}-\mathrm{X}$ set are provided in Figures $\mathrm{S} 1$ and $\mathrm{S} 2$.

a) ELF Basins

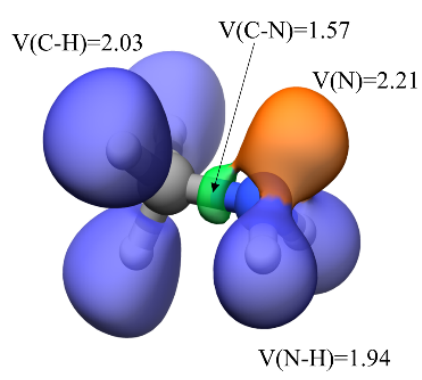

b) Basin Populations

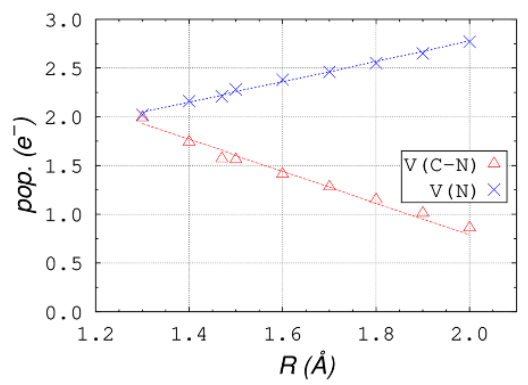

c) Basin Volumes

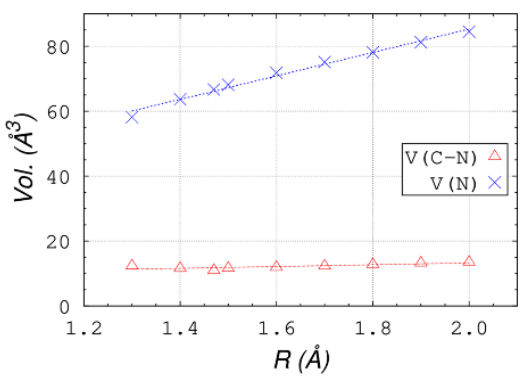

Figure 2. a) $\mathrm{CH}_{3}-\mathrm{X}$ ELF basins (isovalue=0.8) and populations (equilibrium geometry). $\mathrm{V}(\mathrm{C}-\mathrm{H})$ basins are shown in blue, $\mathrm{V}(\mathrm{C}-\mathrm{C})$ and $\mathrm{V}(\mathrm{C}-\mathrm{N})$ in green, and $\mathrm{V}(\mathrm{N})$ in orange. b) $\mathrm{V}(\mathrm{C}, \mathrm{N})$ and $\mathrm{V}(\mathrm{N})$ populations as a function of the $\mathrm{C}-\mathrm{N}$ bond distance; $\mathrm{R}^{2}=0.982$ for $\mathrm{V}(\mathrm{C}, \mathrm{N})$ and 0.989 for $\mathrm{V}(\mathrm{N})$. c) $\mathrm{V}(\mathrm{C}, \mathrm{N})$ and $\mathrm{V}(\mathrm{N})$ volumes as a function of the $\mathrm{C}-\mathrm{N}$ bond distance; $\mathrm{R}^{2}=0.631$ for $\mathrm{V}(\mathrm{C}, \mathrm{N})$ and 0.988 for $\mathrm{V}(\mathrm{N})$.

In the following sections, interacting potentials for the kinetic, electrostatic and exchange correlation terms involving the $\mathrm{C}-\mathrm{X}$ bond and lone pairs (valence) will be developed from the ELF-IQA partition. In all cases, the difference between the calculated IQA energy and the DFT obtained one $(\Delta E)$ was used to evaluate integral accuracy. The error held roughly constant and lower than $1 \%$ of the total energy, as shown in Supporting Information, Table S1.

\section{Classical electrostatic interaction energy}

Since ELF basins are non-overlapping, the electrostatic interaction between them can be easily approximated by the coulombic interaction between single charges located at the barycenter of the basin charge density. We have carried out the test on the reference molecule, $\mathrm{CH}_{3} \mathrm{NH}_{2}$. The center of charge of the $\mathrm{C}-\mathrm{N}$ bond only deviates 0.07 a.u. from the ELF attractor. This small difference is clearly negligible when compared to the bond length, 2.75 a.u. Hence, monopole electrostatics can be expected to reproduce reasonably well the electrostatic interactions, that is:

$$
E_{\text {elec }}^{A B} \propto \frac{q^{A} \cdot q^{B}}{R}
$$

Where $q^{i}$ refers to the charge of the electron pair $i$ (be it core, bond or lone pair). Hence, the overall electrostatic interaction should follow the classical interacting potential $E_{\text {elec }}=B / R+C$, where $B$ and $C$ are constants. For testing this hypothesis, we used ELFIQA to calculate the classical component of $E_{\text {intra }}$ and $E_{\text {inter }}$ (see eqs. 6 and 7 ) for interactions between $\mathrm{C}-\mathrm{X}$ bond $(\mathrm{V}(\mathrm{C}, \mathrm{X}))$ and both, $\mathrm{C}$ and $\mathrm{X}(\mathrm{C}(\mathrm{C})$ and $\mathrm{C}(\mathrm{X}))$, as well as interactions between $\mathrm{X}$ lone pairs $(\mathrm{V}(\mathrm{X}))$ and $\mathrm{X}(\mathrm{C}(\mathrm{X}))$. Then, we represented these contributions against $1 / R$. Results for the interaction of valence basins in methanamine are 
shown in Figure 3 (see Figure S3 for results in all other molecules). As it can be seen, the linear regression fittings present very good correlation coefficients, with $R^{2}$ being higher than 0.97 in all molecules, for both, intra and inter-basin contributions (see Figure S3). The good agreement confirms that the ELF maximum can be used to approximate a point charge behaviour, as well as the validity of eq 8 for intra-basin electrostatic energy. The electrostatic interaction inside a given basin is a bit more difficult to rationalize. Since chemical reorganizations affect the valence, this is specially relevant for bonds and lone pairs. Assuming a homogeneous electron gas-like distribution within electron pairs, it can be shown that a relationship similar to point charges holds (see S.I. for the development).

Agreement is also maintained when charges are taken into account (see Figure S4). Overall, this indicates that we are facing a partition (ELF) which is meaningful at zero order from the electrostatic point of view.

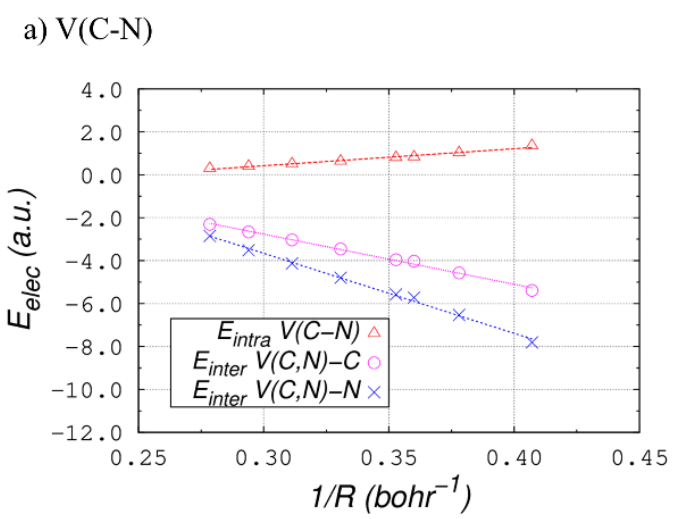

b) $\mathrm{V}(\mathrm{N})$

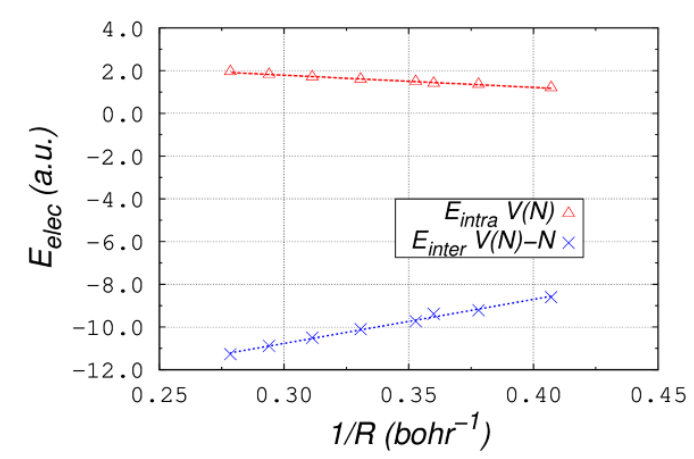

Figure 3. ELF-IQA computed classical electrostatic interaction energy (intra and inter) against $1 / R$ for a) $\mathrm{V}(\mathrm{C}-\mathrm{N})$ and, b) $\mathrm{V}(\mathrm{N})$ in the methanamine molecule. Regression coefficients: $R^{2}=0.978$ for $E_{\text {intra }} \mathrm{V}(\mathrm{C}, \mathrm{N})$, 0.995 for $E_{\text {inter }} \mathrm{V}(\mathrm{C}-\mathrm{N})-\mathrm{C}, 0.996$ for $E_{\text {inter }} \mathrm{V}(\mathrm{C}-\mathrm{N})-\mathrm{N}, 0.989$ for $E_{\text {intra }} \mathrm{V}(\mathrm{N})$, and 0.994 for $E_{\text {inter }} \mathrm{V}(\mathrm{N})-\mathrm{N}$.

\section{Kinetic energy}

To analyse the evolution of the valence kinetic energy, we can assume that the electron density in the low density region (i.e. bonds far from the core) can be described by a zero order development of the kinetic energy density (homogeneous electron density, $\left.t(r)=c_{F} \rho^{5 / 3}\right)$. If we also take into account the linear evolution of charges upon stretching (Figures $2 \mathrm{~b}$ and $\mathrm{S} 2$ ), we obtain a simple expression for the kinetic energy in valence basins (eq 9) (see S.I. for the development). 


$$
T=\frac{4 \pi q^{5 / 3}}{3} \frac{1}{R_{B}^{2}}
$$

The expression provided in eq 9 depends on the bond basin radius $\left(R_{B}\right)$. In order to verify whether we can relate it with the $\mathrm{C}$-X bond distance, we calculate $R_{B}$ as a function of $R$. For that, we subtracted the core radii ${ }^{41}$ to the interatomic distance. Since results show that $R_{B}$ (and $\left.R_{L P}\right) \propto R$ (see Figure S5), we can develop a working expression of the form: $T \propto R^{-2}$. The representation of the kinetic energy in the valence basins of $\mathrm{CH}_{3} \mathrm{NH}_{2}$, i.e. $\mathrm{V}(\mathrm{C}, \mathrm{N})$ and $\mathrm{V}(\mathrm{N})$ are provided in Figure 4; fittings for other molecules being provided in Supporting Information (Figure S6). Overall, the agreement within the whole series of molecules leads to regression coefficients, $R^{2}$, ranging from 0.984 to 1.00 for both bonds and lone pairs. This result evidences the validity of the previous expression for bond and lone pair kinetic energies.

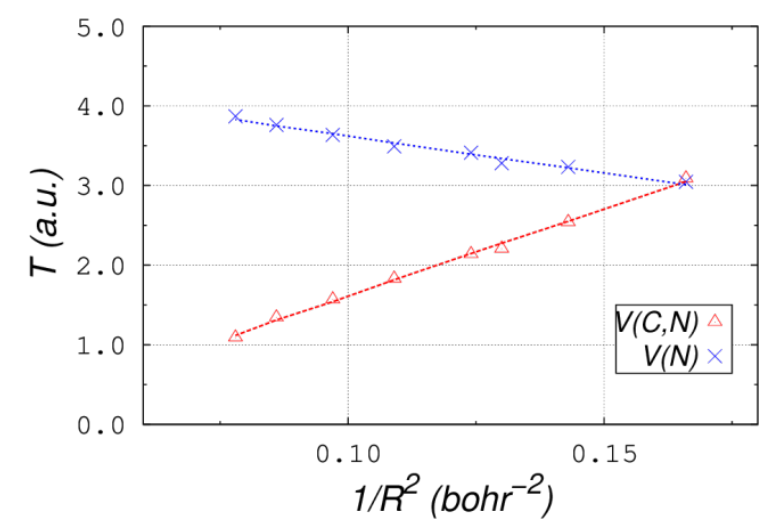

Figure 4. ELF-IQA computed bond kinetic energy against $1 / R^{2}$ for $\mathrm{V}(\mathrm{C}-\mathrm{N})$ and $\mathrm{V}(\mathrm{N})$ basins in $\mathrm{CH}_{3}-\mathrm{NH}_{2}$. Regression coefficients: $R^{2}=0.997$ for $\mathrm{V}(\mathrm{C}, \mathrm{N})$ and 0.984 for $\mathrm{V}(\mathrm{N})$.

\section{Exchange-Correlation energy}

Finally, let us examine the quantum mechanical contributions. ExchangeCorrelation interactions have been shown to follow a linear behaviour: ${ }^{42}$

$$
E_{x-c}^{A B} \approx-\frac{\delta^{A B}}{2 R}
$$

Usually applied to QTAIM atoms, this leads to an exponential decay with distance affected due to $\delta^{A B}$, the delocalization index which depends on the overlap. In the case of ELF partitions, we have found that $\delta^{A B}$ has a linear behaviour $(k / R)$ for interactions between bond and core basins in the range of distances considered (Figure S7). Accordingly, the effective expression for the exchange-correlation energy should follow eq 11. Figure 5 shows the results for the amine, the fittings for all molecules being provided in Figure S8 of the Supporting Information. Very good regression coefficients, $R^{2}>0.99$ in most cases, were obtained, confirming the validity of the previous reasoning and the derived dependencies.

$$
E_{x-c}^{A B} \approx-\frac{\delta^{A B}}{2 R} \approx-\frac{k}{R^{2}}
$$




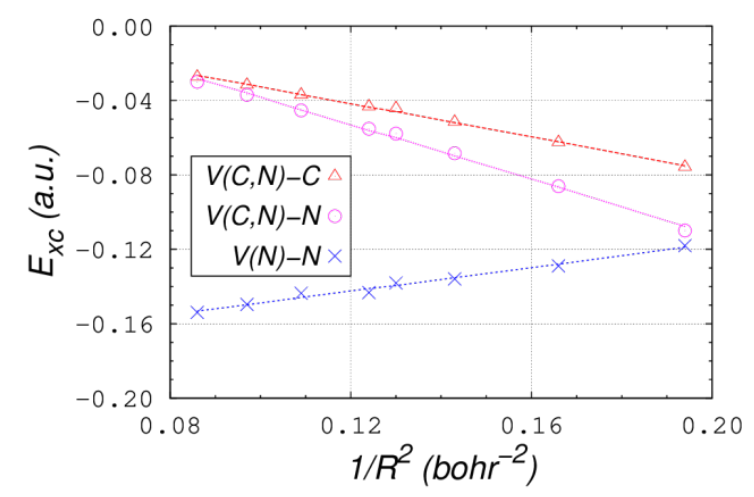

Figure 5. ELF-IQA computed exchange-correlation energies against $1 / R^{2}$ for $\mathrm{V}(\mathrm{C}-\mathrm{N})$ and $\mathrm{V}(\mathrm{N})$ basins with $\mathrm{C}$ and $\mathrm{N}$ in $\mathrm{CH}_{3}-\mathrm{NH}_{2}$. Regression coefficients: $R^{2}=0.998$ for $\mathrm{V}(\mathrm{C}, \mathrm{N})-\mathrm{C}, 0.997$ for $\mathrm{V}(\mathrm{C}, \mathrm{N})-\mathrm{N}$ and 0.988 for $\mathrm{V}(\mathrm{N})-\mathrm{N}$.

\section{Interplay of terms}

Previous results have important consequences that are highly relevant in both force field development and quantum chemical topology. Firstly, it confirms the ability of an electron pair force field (that includes chemical bonds as well as lone pairs) derived from topology to describe the system while leading to a well-behaved quantum contribution. This contribution is also easy to parametrize and to add to "classical" potentials.

Secondly, the quantum mechanical exchange-correlation energy term is found to be noticeably smaller than the classical term for all systems in the dataset (see Figure 6). As an example, we provide the different contributions for methanamine in the equilibrium position. For the $\mathrm{V}(\mathrm{C}, \mathrm{N})$ basins: $T_{V(C, N)}=2.207$ a.u., $E_{\text {elec-intra } \mathrm{V}(\mathrm{C}, \mathrm{N})}=0.825$ a.u., $E_{\text {elec-inter }}$ $V(C, N)-C=-4.028$ a.u., $E_{\text {elec-inter } V(C, N)-N}=-5.723$ a.u., $E_{x-c} V(C, N)-C=-0.044$ a.u., $E_{x-c} V(C, N)-N=-$ 0.058 a.u. For $\mathrm{V}(\mathrm{N}): T_{V(N)}=3.281$ a.u., $E_{\text {elec-intra } V(N)}=1.401$ a.u., $E_{\text {elec-inter } V(N)-N}=-9.379$ a.u., $E_{x-c} V(N)-N=-0.138$ a.u.

It is interesting to note that exchange-correlation terms are two orders of magnitude smaller than classical terms for bonds. Nonetheless, they are only one order of magnitude smaller for the lone pairs. This reveals the greater relevance of quantum effects in the description of lone pairs, which can be neglected for covalent bonds but should be taken into account when developing accurate interacting potentials for lone pairs. Furthermore, this prevalence of quantum effects further justifies the explicit treatment of lone pairs in Force Fields, which are often simply included in atomic contributions. 


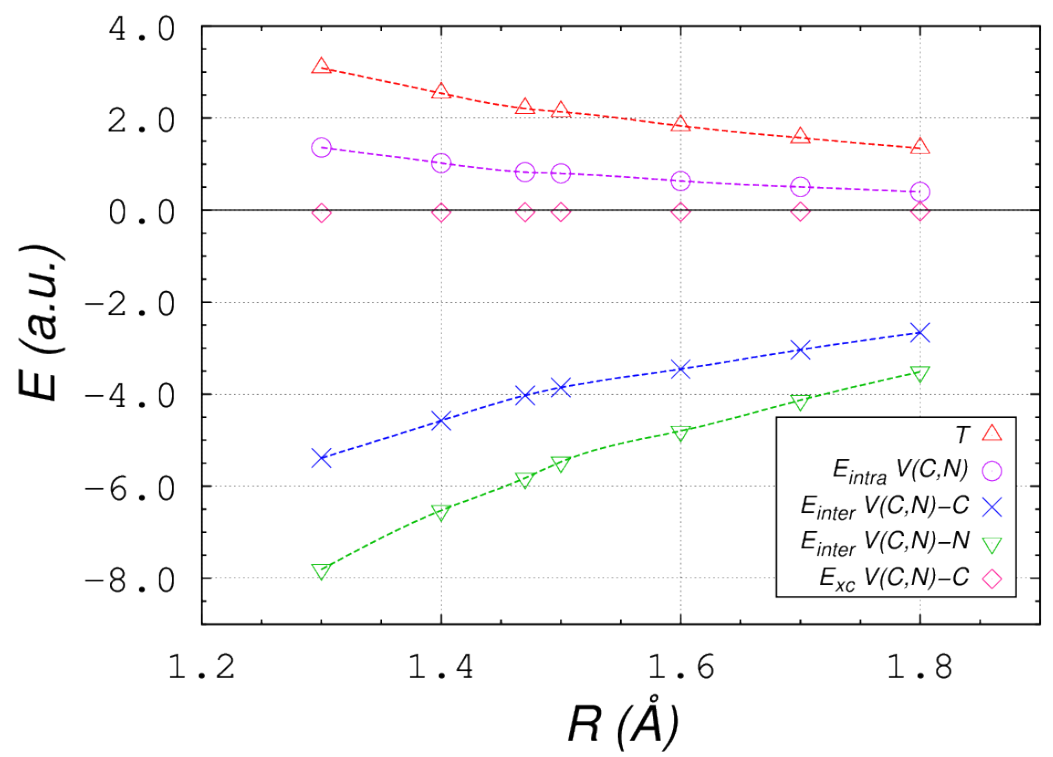

Figure 6. ELF-IQA energy terms for C-N bond in methanamine.

As a final check, Table S3 shows that core energies (kinetic, electrostatic and exchange-correlation) remain constant upon elongation, so that they can be ignored in the construction of the interacting potential.

\section{Comparison with previous models}

Up to now, we have coupled IQA with the ELF topology to obtain the different interaction energy terms involving Lewis entities. According to the previous discussion, the total bond energy would be the sum of kinetic, electrostatic and exchange-correlation contributions, even though in most cases we can neglect this last one. Interestingly, this formulation is formally equivalent to the one provided by the Bond Charge Model (BCM). ${ }^{13}$ This approach is a simple albeit chemically intuitive model for calculating the energy of molecules (or solids). In particular, a homonuclear diatomic molecule, say $A_{2}$, is represented as two cores with $+q / 2$ positive charge, each of them interacting through a bond holding a charge of $-q$ electrons (see Figure 7 top). The bond charge moves along the bond length $\left(R_{B}\right)$, which is a fraction of the equilibrium interatomic distance $(R), R_{B}=v R$. The total energy, which depends on the charge and the bond length, is the sum of three different contributions (eq 12, in atomic units).

$$
E(q, R)=E_{0}+E_{1}+E_{2}=2 E_{A}\left(\frac{q}{2}\right)-C \frac{q^{2}}{R}+\mathrm{D}^{\prime} \frac{q}{R_{B}^{2}}
$$

Where $E_{0}$ is the core energy (equal to $2 E_{A}$ for a homonuclear molecule, with $E_{A}$ being the core energy of each atom), $E_{1}$ accounts for classical electrostatic interactions (core-bond and core-core) and $E_{2}$ represents the kinetic energy of the bond electrons moving along the bond length, $R_{B}$. The model can also be expanded to heteronuclear systems, say $A B$, (see Figure 7 bottom) leading to equation 13. This expression is formally equivalent to the one obtained for homonuclear molecules. 


$$
E=E_{A}+E_{B}-C^{\prime} \frac{q^{2}}{R}+\mathrm{D}^{\prime} \frac{q}{R_{B}^{2}}
$$

Originally, model parameters were obtained by fitting to experimental data. ${ }^{13,14}$ However, it is remarkable that the BCM model has the same energy terms that have been analysed by means of ELF-IQA approach (with the exception of the exchange-correlation term, which is non-classical) and they present the same dependencies with $R$. The parallelism with the terms derived from our ELF-IQA energy model is thus evident:

$$
\begin{gathered}
E_{\text {core }}=E_{A} \\
E_{\text {intra }}^{\text {Bond }}+E_{\text {inter }}^{A-B \text { Bond }}=-C^{\prime} \frac{q^{2}}{R_{B}} \\
T_{\text {Bond }}=D^{\prime} \frac{q}{R_{B}^{2}} \\
E_{x c} \approx 0
\end{gathered}
$$

The first three equations have been shown to work for our test set. As for the exchange-correlation contribution, a comparison of the scales of Figures 3 and 4 provides a first idea of the error. As previously stated, bond exchange-correlation terms are typically two orders of magnitude smaller than the other terms for bonds and one order of magnitude for lone pairs (see Figure 6).
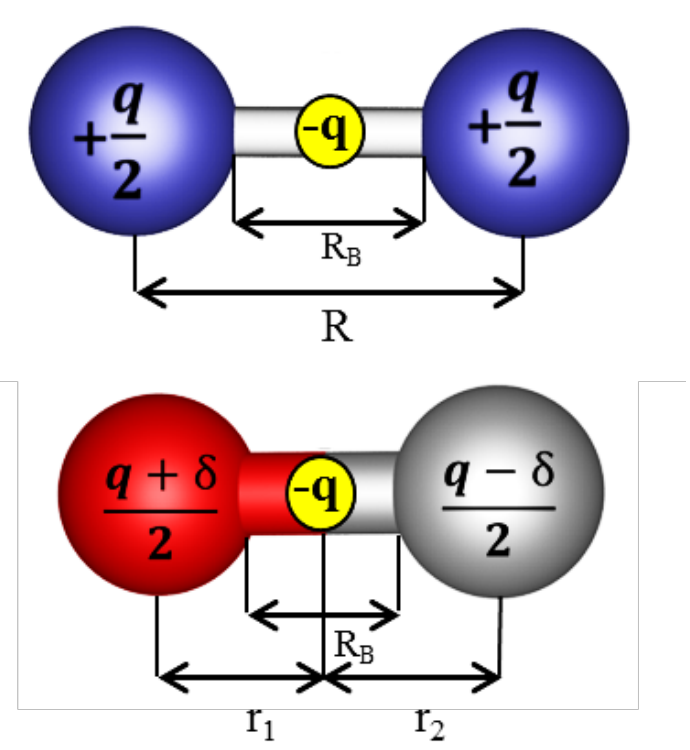

Figure 7. Bond Charge Model representation of a homonuclear molecule (top) and a heteronuclear one (bottom).

It is important to note that this framework explains the previous success of the BCM model, as energy terms with a classical origin are the only ones that contribute significantly to the total energy of molecules where electrons are well-localized. It also justifies the success of ELF parameters linked to this model. ${ }^{43}$ Moreover, it explains why this model was of great success for extended solids in material science, while not so much applied for open (layers, chains) structures, where lone pairs are usually present. As we have seen, neglecting the exchange-correlation terms is straight forward for bonds (two orders of magnitude smaller than kinetic and electrostatic terms), but not so much for lone 
pairs (only one order of magnitude smaller). The next section will be devoted to the analysis of the limits of the IQA-ELF potential.

\section{Limits of the model}

Since the model is based on localized electron pairs, it is perfectly compatible with the description of covalent bonds. Accordingly, it may not suffice to capture other types of interaction. Several examples pertaining to distinct bonding regimes have been studied to estimate the range of applicability. On the basis of electron pair localization, it should be possible to predict the outlier cases where the IQA-ELF potential is not valid. The following systems have been addressed: $\mathrm{BH}_{3}-\mathrm{NH}_{3}, \mathrm{BeH}_{2}-\mathrm{NH}_{3}, \mathrm{CH}_{3}-\mathrm{Li}$ and $\mathrm{Li}_{2}$, as paradigmatic examples of dative covalent bond, non-covalent beryllium bond, ionic and metallic systems (see Figure 8). ${ }^{44,45}$
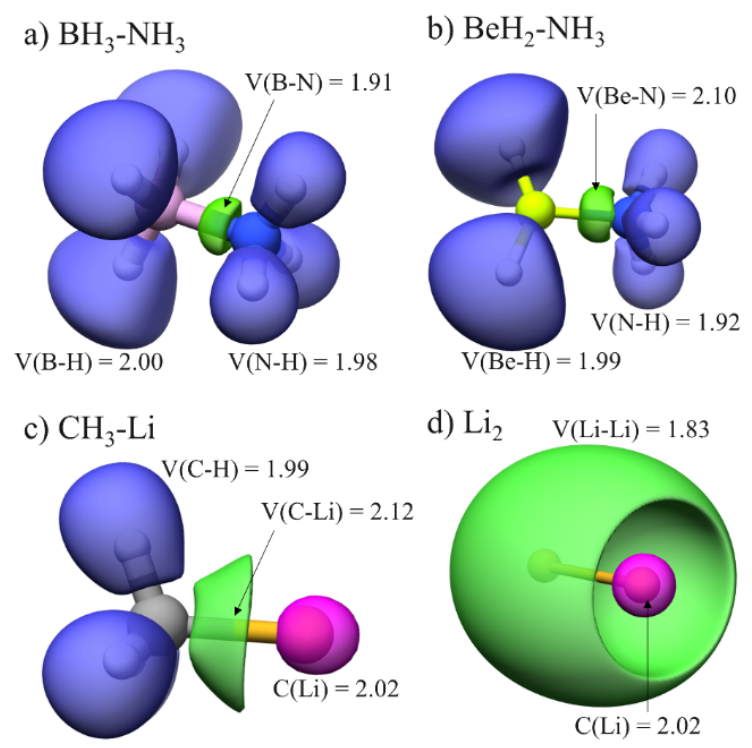

Figure 8. ELF basins (isovalue=0.8) and populations for a) $\mathrm{BH}_{3}-\mathrm{NH}_{3}$, b) $\mathrm{BeH}_{2}-\mathrm{NH}_{3}$, c) $\mathrm{CH}_{3}-\mathrm{Li}$, and d) $\mathrm{Li}_{2}$. Hydrogenoid, disynaptic and core basins are shown in blue, green and purple respectively.

It was found that both dative and very polar bonds still follow the proposed dependencies for the IQA-ELF potential. Specifically, $\mathrm{BH}_{3}-\mathrm{NH}_{3}$ and $\mathrm{BeH}_{2}-\mathrm{NH}_{3}$ molecules, lead to linear regression coefficients, $R^{2}$, for the classical, exchange-correlation, and bond kinetic energies higher than 0.97 for $\mathrm{BH}_{3}-\mathrm{NH}_{3}$ and 0.99 for $\mathrm{BeH}_{2}-\mathrm{NH}_{3}$ (see tables $\mathrm{S} 3$ and S4). The exchange-correlation contribution was also negligible in comparison with the classical energy terms, being two orders of magnitude lower, as in the $\mathrm{CH}_{3}-\mathrm{X}$ series. Similar results are obtained for $\mathrm{CH}_{3}-\mathrm{Li}$. Interestingly, the $\mathrm{C}(\mathrm{Li})-\mathrm{V}(\mathrm{C}-\mathrm{Li})$ electrostatic term is smaller than the $\mathrm{C}(\mathrm{C})-\mathrm{V}(\mathrm{C}-\mathrm{Li})$ counterpart by almost one order of magnitude. This result suggests that this contribution is not energetically meaningful, which can be related with the partially ionic character of the bond, which leads to the bonding basin being much closer to the carbon than to the lithium core $(0.829 \AA$ for $\mathrm{C}$ vs $1.169 \AA$ for Li).

The proposed behavior of the energy terms does not hold for $\mathrm{Li}_{2}$. From the visual point of view, a rather remarkable feature is already identifiable in Figure $8 \mathrm{~d}$. The volume of the bond (where a non-nuclear maximum of the density appears) is much bigger than in 
previous cases ( 898.23 vs $17.91 \AA^{3}$ in ethane). Moreover, the population in this basin also follows a different behavior upon stretching. It remains constant (between 1.81 and $1.83 \mathrm{e}^{-}$) independently of the Li-Li interatomic distance. The resulting ELF-IQA kinetic, electrostatic and exchange-correlation energy terms are shown in Figure 9. It is noteworthy that all the terms are very small, of the order of $10^{-4}$ a.u., unlike the ones obtained for $\mathrm{CH}_{3}-$ $\mathrm{X}$ molecules, which were of the order of $10^{1}$ a.u. (kinetic and electrostatic terms) and $10^{-1}$ a.u. (exchange-correlation term). We can thus consider that the model works properly for all the molecules considered but $\mathrm{Li}_{2}$, for which the model is not suitable.

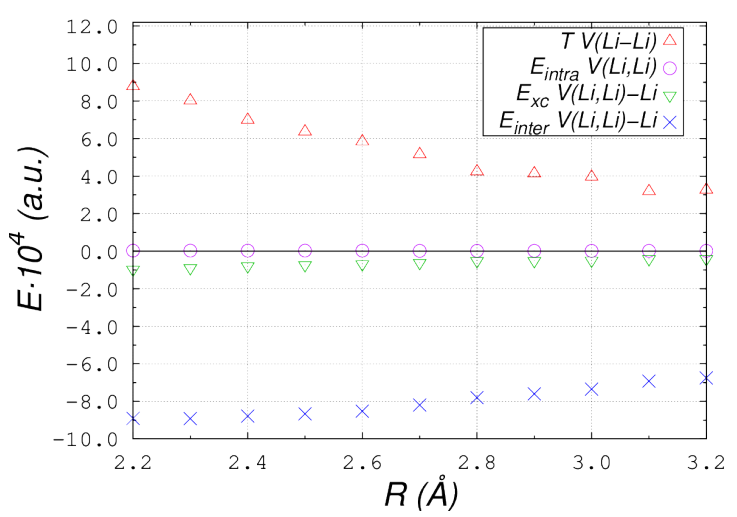

Figure 9. Kinetic, classical (intra and inter) and exchange-correlation energy terms for Li-Li bond in $\mathrm{Li}_{2}$. For simplicity, energy values are multiplied by $10^{4}$.

Overall, the model is shown to be valid for partial covalency, while failing for metallic bonds (as the case of $\mathrm{Li}_{2}$ ). It should be noted that these two categories can be easily identified from properties derived from the electron density. Over the years, numerous attempts have been made to provide a distinct classification of the chemical bond from topological characteristics, ${ }^{46,47}$ with special emphasis on the metallic bond. ${ }^{48-50}$ Complex descriptors, as the metallicity index $\left(\xi_{m}\right)$ proposed by Ayers et al..$^{50}$ have been used to further collect the character of bonds though topological measurements. This index is constructed to highlight regions of planar density, its expression being provided in eq. 14.

$$
\xi_{m}\left(r_{b c p}\right)=\frac{36\left(3 \pi^{2}\right)^{2 / 3}}{5} \frac{\rho\left(r_{b c p}\right)^{5 / 3}}{\nabla^{2} \rho\left(r_{b c p}\right)}
$$

High values of $\xi_{m}$ are indicative of metallic character (typically $\xi_{m}>25$ in solids), while weak metallic bonds are identified by $\xi_{m}$ values between 1 and 5 . When $\xi_{m}$ is inferior to 1 , the bonds present a non-metallic character. Bonds of all the previous molecules were characterized through their electron density, $\rho(r)$, and its Laplacian, $\nabla^{2} \rho(r)$ and the aforementioned derivative descriptor at the bond critical point. Given that local topological indices extrapolate the characteristics of a single point to a large chemical entity, a larger basis set has been used to guarantee converged densities and robustness. Results are presented in Table 1.

Table 1. Topological characteristics of the selected bonds. Calculations performed at the B3LYP/6$311 \mathrm{G}(d, p)$ level.

\begin{tabular}{cccc}
\hline Bond & $\rho_{B C P}(\mathrm{a} . \mathrm{u})$ & $\nabla^{2} \rho_{B C P}(\mathrm{a} . \mathrm{u})$ & $\xi_{\mathrm{m}, \mathrm{BCP} *}$ \\
\hline C-C & 0.2378 & -0.5315 & -5.5182
\end{tabular}




\begin{tabular}{cccc} 
C-N & 0.2611 & -0.6700 & -5.1158 \\
C-O & 0.2556 & -0.5042 & -6.5602 \\
C-F & 0.2332 & -0.0217 & -130.5568 \\
B-N & 0.0993 & 0.4195 & 1.6314 \\
Be-N & 0.0569 & 0.3374 & 0.8017 \\
C-Li & 0.0438 & 0.2128 & 0.8205 \\
Li- $-\mathrm{Li}$ & 0.0134 & 0.0019 & 12.7803 \\
$* \xi_{\mathrm{m}, \mathrm{BCP}}$ is not defined when $\nabla^{2} \rho_{B C P}<0$, yet it can be calculated to exemplify the significant difference \\
\multicolumn{5}{c}{ between bonds. }
\end{tabular}

First of all, it is clear that the limiting cases we chose lead indeed to positive Laplacians and larger values of $\xi_{m}$. The metallicity index for $\mathrm{Li}_{2}$ is much higher than for the rest of the essayed bonds, which correlates with the breakdown of our model. Furthermore, the results manage to partially justify the lesser degree of accuracy of the model for the B-N, Be-N and C-Li bonds, as they exhibit greater $\xi_{m}$ indices and positive Laplacian values at their BCP. This feature can be related to their only-partial covalent nature. This leads to a parametrizable model for a very intuitive idea, for those cases where the charge is rather indistinctly delocalized over the system, point charges become a deficient model. Instead, bonds that are assigned as covalent by the charge accumulation found in their BCP can be assumed to be properly described by the model hereby presented.

\section{Conclusions and outlook}

We have presented a simple energy model based on the ELF topology for the stretching of bonds that explains the interaction between electron pairs while retaining their mechanoquantical character. Kinetic, electrostatic and exchange-correlation terms have been shown to follow simple dependencies that are akin to simple energy models. Electron pairs, as described by the Electron Localization Function, can be treated from homogeneous electron gas point of view. These models have been shown to work for different polarities (from homonuclear to highly polar bonds, including non-conventional bonds). Due to the failure of the electron-pair model, its applicability is limited for metallic bonds and can be expected to fail in highly delocalized bonding regimes. Simple local measures have been shown to identify the situations in which the model is not valid.

Moreover, the energy model hereby introduced is able to explain the success of simple previous models, such as the Bond Charge Model, and its updated version the ELFBCM model. ${ }^{8}$ In these models, the quantum nature of electrons was neglected. Our test set shows that, in general, this contribution (represented by the exchange-correlation term) can indeed be neglected for bonds. Quantum contributions become more important in delocalized electrons, such as lone pairs. As lone pairs are of significant importance in many chemical systems that are usually modelled with Force Field approaches, we hereby justify the careful consideration of their position and electrostatic behavior. The definition of explicit lone pairs has already been considered in molecular mechanics modelling, be it mathematically or conceptually. ${ }^{51,52}$

Notably, the development of such a model may have important implications in other closely related fields, as conceptual DFT (c-DFT). ${ }^{53}$ The existence of a simple equation for the system energy would allow to obtain molecular properties 
(electronegativity, chemical hardness, etc.) in a simple manner and starting from chemically meaningful concepts. ${ }^{54,55}$ As previously stated, another field on which this energy model may suppose a breakthrough is in Force Field development. In this respect, our model provides useful insight on non-classical terms and further justifies the design of Force Fields transcending the all-atom paradigm. Such Force Fields have already achieved remarkable success, ${ }^{56}$ and we expect to foster these novel modelling methods. The work herein presented also opens the doors towards the further development on energy models based on Quantum Chemical Topology.

\section{References}

(1) Allinger, N. L. Adv. Phys. Org. Chem. 1976, 13, 1-82.

(2) McAliley, J. H.; Bruce, D. A. J. Chem. Theory Comput. 2011, 7 (11), 3756-3767.

(3) Wang, M.; Hu, X.; Beratan, D. N.; Yang, W. J. Am. Chem. Soc. 2006, 128 (10), 3228-3232.

(4) Morokuma, K. J. Chem. Phys. 1971, 55 (3), 1236-1244.

(5) Ziegler, T.; Rauk, A. Theor. Chim. Acta 1977, 46 (1), 1-10.

(6) Bader, R. F. W. Atoms in molecules : a quantum theory; Clarendon Press, 1990.

(7) Contreras-García, J.; Marqués, M.; Menéndez, J.; Recio, J. Int. J. Mol. Sci. 2015, 16 (4), 8151-8167.

(8) Contreras-García, J.; Cardenas, C. J. Mol. Model. 2017, 23 (9), 271.

(9) Johnson, M. A. Nat. Chem. 2009112009.

(10) Gillespie, R. J.; Nyholm, R. S. Q. Rev. Chem. Soc. 1957, 11 (4), 339.

(11) Ekesan, S.; Kale, S.; Herzfeld, J. J. Comput. Chem. 2014, 35 (15), 1159-1164.

(12) Herzfeld, J.; Ekesan, S. Phys. Chem. Chem. Phys. 2016, 18 (44), 30748-30753.

(13) Parr, R. G.; Borkman, R. F. J. Chem. Phys. 1968, 49 (3), 1055-1058.

(14) Borkman, R. F.; Parr, R. G. J. Chem. Phys. 1968, 48 (3), 1116-1126.

(15) Pendás, A. M.; Blanco, M. A.; Francisco, E. J. Chem. Phys. 2004, 120 (10), 45814592.

(16) Maxwell, P.; Pendás, Á. M.; Popelier, P. L. A. Phys. Chem. Chem. Phys. 2016, 18 (31), 20986-21000.

(17) Syzgantseva, O. A.; Tognetti, V.; Joubert, L. J. Phys. Chem. A 2013, 117 (36), 8969-8980.

(18) Guevara-Vela, J. M.; Chávez-Calvillo, R.; García-Revilla, M.; Hernández-Trujillo, J.; Christiansen, O.; Francisco, E.; Martín Pendás, Á.; Rocha-Rinza, T. Chem. - A Eur. J. 2013, 19 (42), 14304-14315.

(19) Munarriz, J.; Velez, E.; Casado, M. A.; Polo, V. Phys. Chem. Chem. Phys. 2018, 20 (2), 1105-1113.

(20) Popelier, P. L. A. Int. J. Quantum Chem. 2015, 115 (16), 1005-1011.

(21) Di Pasquale, N.; Davie, S. J.; Popelier, P. L. A. J. Chem. Theory Comput. 2016, 12 (4), 1499-1513.

(22) Zielinski, F.; Maxwell, P. I.; Fletcher, T. L.; Davie, S. J.; Di Pasquale, N.; Cardamone, S.; Mills, M. J. L.; Popelier, P. L. A. Sci. Rep. 2017, 7 (1), 12817.

(23) Savin, A.; Nesper, R.; Wengert, S.; Fässler, T. F. Angew. Chemie Int. Ed. English 1997, 36 (17), 1808-1832.

(24) Martín Pendás, A.; Francisco, E.; Blanco, M. A. Chem. Phys. Lett. 2008, 454 (4-6), 396-403.

(25) Parr, R. G.; Borkman, R. F. J. Chem. Phys. 1968, 49 (3), 1055-1058.

(26) Frisch, M. J.; Trucks, G. W.; Schlegel, H. B.; Scuseria, G. E.; Robb, M. A.; 
Cheeseman, J. R.; Scalmani, G.; Barone, V.; Mennucci, B.; Petersson, G. A.; Nakatsuji, H.; Caricato, M.; Li, X.; Hratchian, H. P.; Izmaylov, A. F.; Bloino, J.; Zheng, G.; Sonnenberg, J. L.; Hada, M.; Ehara, M.; Toyota, K.; Fukuda, R.; Hasegawa, J.; Ishida, M.; Nakajima, T.; Honda, Y.; Kitao, O.; Nakai, H.; Vreven, T.; Montgomery Jr., J. A.; Peralta, J. E.; Ogliaro, F.; Bearpark, M.; Heyd, J. J.; Brothers, E.; Kudin, K. N.; Staroverov, V. N.; Kobayashi, R.; Normand, J.; Raghavachari, K.; Rendell, A.; Burant, J. C.; Iyengar, S. S.; Tomasi, J.; Cossi, M.; Rega, N.; Millam, J. M.; Klene, M.; Knox, J. E.; Cross, J. B.; Bakken, V.; Adamo, C.; Jaramillo, J.; Gomperts, R.; Stratmann, R. E.; Yazyev, O.; Austin, A. J.; Cammi, R.; Pomelli, C.; Ochterski, J. W.; Martin, R. L.; Morokuma, K.; Zakrzewski, V. G.; Voth, G. A.; Salvador, P.; Dannenberg, J. J.; Dapprich, S.; Daniels, A. D.; Farkas, Ö.; Foresman, J. B.; Ortiz, J. V; Cioslowski, J.; Fox, D. J. .

(27) Lee, C.; Yang, W.; Parr, R. G. Phys. Rev. B 1988, 37 (2), 785-789.

(28) Becke, A. D. J. Chem. Phys. 1993, 98 (7), 5648-5652.

(29) Dunning, T. H. J. Chem. Phys. 1971, 55 (2), 716-723.

(30) Dunning, T. H.; Peterson, K. A.; Wilson, A. K. J. Chem. Phys. 2001, 114 (21), 9244-9253.

(31) Perdew, J. P.; Burke, K.; Ernzerhof, M. Phys. Rev. Lett. 1996, 77 (18), 3865-3868.

(32) Becke, A. D. J. Chem. Phys. 1993, 98 (2), 1372-1377.

(33) Adamo, C.; Barone, V. J. Chem. Phys. 1999, 110 (13), 6158.

(34) Peterson, K. A.; Dunning, T. H. J. Chem. Phys. 2002, 117 (23), 10548-10560.

(35) Savin, A.; Jepsen, O.; Flad, J.; Andersen, O. K.; Preuss, H.; von Schnering, H. G. Angew. Chemie Int. Ed. English 1992, 31 (2), 187-188.

(36) Noury, S.; Krokidis, X.; Fuster, F.; Silvi, B. Comput. Chem. 1999, 23 (6), 597-604.

(37) Pettersen, E. F.; Goddard, T. D.; Huang, C. C.; Couch, G. S.; Greenblatt, D. M.; Meng, E. C.; Ferrin, T. E. J. Comput. Chem. 2004, 25 (13), 1605-1612.

(38) Goddard, T. D.; Huang, C. C.; Ferrin, T. E. J. Struct. Biol. 2007, 157 (1), 281-287.

(39) Blanco, M.A., Martín Pendás, A., A.; Francisco, E. 2005.

(40) Pendás, A. M.; Francisco, E.; Blanco, M. A. J. Comput. Chem. 2005, 26 (4), $344-$ 351.

(41) Kohout, M.; Savin, A. Int. J. Quantum Chem. 1996, 60 (4), 875-882.

(42) Francisco, E.; Menéndez Crespo, D.; Costales, A.; Martín Pendás, Á. J. Comput. Chem. 2017, 38 (11), 816-829.

(43) Contreras-García, J.; Marqués, M.; Menéndez, J. M.; Recio, J. M. Int. J. Mol. Sci. 2015, 16 (4), 8151-8167.

(44) Dreux, K. M.; McNamara, L. E.; Kelly, J. T.; Wright, A. M.; Hammer, N. I.; Tschumper, G. S. J. Phys. Chem. A 2017, 121 (31), 5884-5893.

(45) Yáñez, M.; Sanz, P.; Mó, O.; Alkorta, I.; Elguero, J. J. Chem. Theory Comput. 2009, 5 (10), 2763-2771.

(46) Cremer, D.; Kraka, E. Angew. Chemie Int. Ed. English 1984, 23 (8), 627-628.

(47) Bader, R. F. W.; Essén, H. J. Chem. Phys. 1984, 80 (5), 1943-1960.

(48) Mori-Sánchez, P.; Pendás, A. M.; Luaña, V. J. Am. Chem. Soc. 2002, 124 (49), 14721-14723.

(49) Gervasio, G.; Bianchi, R.; Marabello, D. Chem. Phys. Lett. 2004, 387 (4-6), 481484.

(50) Ayers, P. W.; Jenkins, S. Comput. Theor. Chem. 2015, 1053, 112-122.

(51) Lii, J.-H.; Allinger, N. L. J. Phys. Chem. A 2008, 112 (46), 11903-11913.

(52) Oroguchi, T.; Nakasako, M. Sci. Rep. 2017, 7 (1), 15859.

(53) Geerlings, P.; De Proft, F.; Langenaeker, W. Chem. Rev. 2003, 103 (5), 1793-1874.

(54) Geerlings, P.; De Proft, F. Phys. Chem. Chem. Phys. 2008, 10 (21), 3028. 
(55) Balawender, R.; Lesiuk, M.; De Proft, F.; Geerlings, P. J. Chem. Theory Comput. 2018, 14 (2), 1154-1168.

(56) Bai, C.; Herzfeld, J. ACS Cent. Sci. 2016, 2 (4), 225-231. 